\title{
Effect of grinding and extraction conditions on the determination of antioxidant activity and phenolic acids in barley
}

\author{
Bing Zhou ${ }^{1} \cdot$ Zhao Jin $^{2} \cdot$ Paul B. Schwarz ${ }^{2} \cdot$ Yin $i^{3}$ (D)
}

Received: 24 February 2021 / Accepted: 17 May 2021 / Published online: 23 May 2021

(c) The Author(s) 2021

\begin{abstract}
In the present study, the objective was to evaluate the effects of barley kernel size, grind level, and extract solvent on the antioxidant activities associated with total phenolic content and phenolic acid compositions. Three barley varieties (Kindred, Azure, and Tradition), were used and the results showed that with the exception of superoxide anion radical scavenging activity, thin kernel size fraction $(2.0 \mathrm{~mm})$ showed much higher antioxidant activities and total phenolic content than those in the $2.4 \mathrm{~mm}$ and $2.8 \mathrm{~mm}$ fractions when averaged across barley cultivar, grind level, and extract solvent. A similar trend was found for individual phenolic acid compositions among kernel sizes. Simple correlation analysis revealed that total phenolic content showed strong correlation with DPPH· scavenging activity, ABTS + scavenging activity, and reducing power. Generally, fine grind and $80 \%$ acetone extract showed averaged highest values in antioxidant activities, total phenolic content, and individual phenolic acid compositions. Stepwise linear regression showed that extract solvent was the most important factor for DPPH· scavenging activity, ABTS + scavenging activity, reducing power, and iron chelating activity. The sum of syringic acid and caffeic acid exhibited a dominant role in explaining the major variation in antioxidant activities except for superoxide anion radical scavenging activity.
\end{abstract}

Keywords Antioxidant activity $\cdot$ Barley $\cdot$ Kernel size $\cdot$ Milling $\cdot$ Phenolic acids $\cdot$ Stepwise regression

\section{Introduction}

Barley (Hordeum vulgare), an annual cereal grain, is one of the oldest cultivated cereal grains. In decreasing order, it is used worldwide as an animal feed, in the production of malt for beer and distilled spirits, and for food. Epidemiological studies have suggested that increased consumption

Yin Li

Yin.Li@malteurop.com

Bing Zhou

zb35@sohu.com

Zhao Jin

zhao.jin@ndsu.edu

Paul B. Schwarz

paul.schwarz@ndsu.edu

1 Department of Applied Engineering, Zhejiang Economic and Trade Polytechnic, Hangzhou 310018, China

2 Department of Plant Sciences, North Dakota State University, P.O. 5051, Fargo, ND 58105-5051, USA

3 Malteurop North America, Milwaukee, WI 53215, USA of whole grains, and whole grain food is associated with reduced risks of chronic diseases such as cardiovascular disease [1, 2], type II diabetes [3, 4], and certain cancers [5, 6]. Other health benefits from whole grain phytochemicals were reviewed by Okarter and Liu [7].

Whole grains contain not only nutrients such as starch, proteins, fats, etc., but many bioactive phytochemicals such as phenolic compounds, phytosterols, dietary fibers (mainly beta-glucan in barley), carotenoids, tocols, tocopherols, alkylresorinols, lignans, etc. [8-11]. Phenolic compounds in grains act as growth regulators during plant metabolism and exhibit antioxidant capabilities to scavenge free radicals and active oxygen, generate relatively stable phenoxyl radicals, function as reducing agents and chelate metal ions $[8,9]$. Since these bioactive compounds do not present in grain parts (e.g. endosperm, germ and bran) uniformly present in grain tissues (e.g. endosperm, germ, and bran), it is suggested to consume the whole grain as food resources [12]. Research has also indicated that the functional effects of whole grain in human health may be due to the comprehensive effects of nutrients and bioactive phytochemicals in whole grain or foods from 
whole grain [13-15]. The updated 2015 USDA Dietary Guidelines also recommended that Americans increase consumption of whole grains as part of a health-promoting diet [16].

Several factors are known to impact the level of antioxidant activity and phenolics in barley. Zhou et al. [17] recently reported total phenolic content (TPC) and some of the antioxidant activities were significantly impacted by genotype and growing environment. However, in some cases, there were interactions between genotype and environment. Similar results were previously reported for Indian barley by Narwal [18]. Besides, it is known that the outer layers of barley kernels, such as the husk, pericarp, and aleurone, contain much higher levels of phenolic compounds than those of the endosperm [19]. Madhujith et al. [20] confirmed that the outermost fractions yielded the highest phenolic content in two barley samples evaluated. Higher content of phenolic compounds was also observed in wheat bran samples during the studies to compare the antioxidant level in bran with endosperm fractions [21, 22].

Aside from health benefits, phenolic compounds in barley have been shown to have significant influences on beer production and quality. The source of phenolics in beer is either from malted barley or hops, with about $3 / 4$ of total polyphenols coming from barley [19]. Phenolic compounds exhibiting antioxidant and antiradical activities include phenolic acids, flavonoids, proanthocyanidins, tannins and amino phenolic compounds [17, 23, 24]. Phenolic acids can be divided into two groups: hydroxybenzoic acid derivatives (including vanillic, syringic, gallic acids, etc.) and hydroxycinnamic acid derivatives (including ferulic, p-coumaric, caffeic, sinapic acids, etc.) [25].

While there has been limited study with barley, the methods of preparation and extraction of samples are known to impact the determination of phenolics in wheat [26]. Fine grinding of wheat bran has been shown to increase antioxidant activity, and the levels of phenolic acids, flavonoids and anthocyanins. The effect may be attributed to the increased surface area of finer particles. The extraction solvent has also been shown to have an impact. Zhao et al. [27] compared the extract solvent effects on barley antioxidant activities and reported that the highest antioxidant activities were found in $80 \%$ acetone extracts. Similar results have been reported for other plant materials including fruits [28], vegetables [29], legumes [30, 31], and wheat bran [32].

The objective of this study, which has implications for barley utilized in food and beverages, was to investigate the combined effects of barley variety, kernel size, grind particle size and extraction solvent on the antioxidant activity and corresponding phenolic acid composition. And the stepwise regression analysis was used to determine the contribution of individual phenolic acids to the antioxidant activity.

\section{Materials and methods}

\section{Chemicals}

2,2-diphenyl-1-picrylhydrazyl (DPPH), 2,2'-azinobis(3ethylbenzothiazoline-6-sulfonic acid) diammonium salt (ABTS), 6-hydroxy-2,5,7,8-tetramethylchroman carboxylic acid (Trolox), 3-(2-Pyridyl)-5,6-diphenyl-1,2,4triazine-4',4"-disulfonic acid monosodium salt (ferrozine), xanthine oxidase (XOD), nitrotetrazolium blue chloride (NBT), and hypoxanthine (HPX) were purchased from Sigma-Aldrich (St. Louis, MO). Folin-Ciocalteu's phenol reagent was purchased from MP Biomedicals, Inc. (Lot No. 1047J, Solon, OH). All other solvent was purchased from VWR International (West Chester, PA) and ACS grade. All standards were HPLC grade.

\section{Barley samples}

Three barley cultivars utilized in the current study were Azure, Kindred, and Tradition. And they were grown at the North Dakota State University Agricultural Experiment Station in Fargo, ND in two crop years. All were recommended six-rowed malting types. The average protein contents of the three samples ranged from 13.3 to $15.5 \%$. The average plump kernels for Azure, Kindred, and Tradition were $83.9,44.8$, and $55.0 \%$ respectively, while thin kernels averaged $4.4 \%, 54.7 \%$, and $44.0 \%$, respectively.

Each barley sample was divided into three fractions based upon kernel size using a Eureka-Niagara Sample Barley grader (S. Howes Co. Inc., Silver Creek, NY). These were the kernel fractions retained on $2.8 \times 19 \mathrm{~mm}$, $2.4 \times 19 \mathrm{~mm}$, and $2.0 \times 19 \mathrm{~mm}$ slotted sieves. These will subsequently be referred to as the $2.8,2.4$ and $2.0 \mathrm{~mm}$ fractions.

\section{Milling}

Barley Samples were ground on three different mills to provide a range in particle size distribution. The finest particle size (fine grind) was obtained with a Udy mill (Udy, Corp., Fort Collins, CO) fitted with a $0.5 \mathrm{~mm}$ screen. The coarsest particle size (coarse grind) was obtained with a Romer Mill (Romer Labs, Inc., Union, MO) set at the smallest setting. The Perten mill set at 1 (model 3600, Perten Instruments, Huddinge, Sweden) provided a medium grind. The particlesize distribution from the three mills is shown in Table 1, was tested using the American Society of Brewing Chemists method Malt -15. on number 10, 30, 60, 100, 100, and 200 mesh test sieves [33]. 
Table 1 Characterization of particle size in grinds used to determination of antioxidant activities and phenolic acid content in barley

\begin{tabular}{|c|c|c|c|c|c|c|c|}
\hline \multirow[t]{3}{*}{ Grinding } & \multicolumn{7}{|c|}{$\begin{array}{l}\text { Test sieve } \\
\text { (US No. and opening, mm) }\end{array}$} \\
\hline & \multicolumn{7}{|c|}{ (\% of total sample) } \\
\hline & $10(2.00)$ & $30(0.595)$ & $60(0.250)$ & $60(0.250)$ & $120(0.125)$ & $200(0.074)$ & Thru 200 \\
\hline Coarse & 0.0 & 60.7 & 33.7 & 3.2 & 2.8 & 2.7 & 0 \\
\hline Medium & 0.0 & 46.2 & 39.6 & 4.3 & 4.6 & 4.2 & 1.0 \\
\hline Fine & 0 & 6.1 & 60.6 & 24.5 & 6.1 & 2.2 & 0.6 \\
\hline
\end{tabular}

\section{Preparation of extracts from barley}

Ground barley samples $(1 \mathrm{~g})$ were extracted with $20 \mathrm{~mL}$ of $80 \%$ acetone $(\mathrm{v} / \mathrm{v})$ or water under nitrogen on a reciprocating shaker for $1 \mathrm{~h}$ at $20^{\circ} \mathrm{C}$. After centrifugation $(4,000 \mathrm{~g}$, $10 \mathrm{~min}$ ), the supernatant was collected. To avoid oxidation, extracts were stored in the dark at $-20{ }^{\circ} \mathrm{C}$ and analyses were performed within $24 \mathrm{~h}$.

\section{Total phenolic content and antioxidant activities}

All methodology was detailed in our previous publication [17]. Total phenolic content (TPC) was determined according to the method described by $\mathrm{Xu}$ [33]. Radical scavenging activities, using three common radicals, DPPH, ABTS +, and superoxide $(\mathrm{O} 2 \cdot-)$ were determined using methods described by $\mathrm{Xu}$ et al. [31]. Iron (II) chelating activity and reducing power, were determined according to the methods of Xu et al. [31] and Oktay et al. [34], respectively.

\section{HPLC analysis of free phenolic acids}

Prior to HPLC analysis, barley extracts were evaporated to dryness under vacuum at $35^{\circ} \mathrm{C}$ and redissolved in $1.5 \mathrm{~mL}$ of $25 \%$ methanol. The methanol solution was filtered through a $0.45 \mu \mathrm{m} \times 13 \mathrm{~mm}$ acrodisc syringe filter with nylon membrane (Pall Corporation, New York City) and analyzed for free phenolic acid content by HPLC. The quantitative analysis of free phenolic acids was performed according to the method of Yu et al. [35] with slight modifications. A Hewlett Packard 1050 (HP 1050) series HPLC chromatography system equipped with a Photodiode Array Detector (PAD) (Agilent Technologies, Waldbronn, Germany) was used. The detection and quantification were performed at 280 and $325 \mathrm{~nm}$ for phenolic acid composition. A $4.6 \mathrm{~mm} \times 150 \mathrm{~mm}$, $5 \mu \mathrm{m}$, Supelcosil LC-18 column (Sigma, St. Louis, MO), maintained at $30{ }^{\circ} \mathrm{C}$ was used for separation. Elution was performed using mobile phase A ( $0.01 \mathrm{M}$ sodium citrate buffer, $\mathrm{pH}$ 5.4) and mobile phase B (methanol), the solvent flow rate was set at $1.0 \mathrm{~mL} / \mathrm{min}$. The solvent gradient in volumetric ratios was as follows: $0 \mathrm{~min}, 2 \% \mathrm{~B} ; 12 \mathrm{~min}, 4 \%$ B; 20 min, $13 \%$ B; 22 min, 13\% B; 26 min, $2 \%$ B. The solvent gradient was held at $2 \% \mathrm{~B}$ for an additional 6 min and the gradient was increased to $90 \% \mathrm{~B}$ at $33 \mathrm{~min}$. The solvent gradient was held at $90 \%$ B for an additional 10 min to clean the column.

For qualitative identification of HPLC peaks from samples, $1 \mathrm{mg} / \mathrm{mL}$ of stock solution of 12 individual phenolic acid compounds (gallic acid, protocatechuic acid, 4-hydroxybenzoic acid, vanillic acid, caffeic acid, syringic acid, chlorogenic acid, p-coumaric acid, m-coumaric acid, ferulic acid, o-coumaric acid, and sinapinic acid) was prepared and diluted to $100 \mu \mathrm{g} / \mathrm{mL}$. The diluted working solutions were injected into HPLC, and the spiking method and standard external method were used by comparing peak area and retention time. All identified phenolic acids were quantified with external standards by using HPLC analysis as described previously. To prepare $1 \mathrm{mg} / \mathrm{mL}$ of stock solution of standard mixture, $10 \mathrm{mg}$ of each phenolic acid compound were mixed and dissolved into $10 \mathrm{~mL}$ of $25 \%$ methanol. The stock solution was further diluted into standard working solutions $(100,50,25,10,5,2.5,1,0.5,0.25 \mu \mathrm{g} / \mathrm{mL})$ and stored at $-20{ }^{\circ} \mathrm{C}$.

\section{Experimental design and statistical analysis}

A randomized complete block design (RCBD) with duplicate experiments was carried out throughout the study. Replication was accomplished by repeating the experiment once. Barley cultivar, kernel size, grind level, and extract solvent were designated as independent variables (Table 2). Treatment means were considered significantly different at $\mathrm{P} \leq 0.05$.

Statistical analyses were done by using procedures of the Statistical Analysis System (version 9.1, SAS Institute, Cary, NC). Data were analyzed by an analysis of variance (ANOVA) procedure using the general linear model. Tukey's pairwise comparison test was used to determine the significant differences $(P \leq 0.05)$. Means were separated using the least significant differences (LSD).

The GLM was used to perform a forward stepwise regression with $\alpha$-to-enter $=0.15$ and $\alpha$-to-remove $=0.15$ [36]. Stepwise regression was used to determine how much variability could be explained by each independent variable (e.g., barley cultivar, kernel size fractions, ground level, and extract solvent) for the dependent variables (e.g., antioxidant 


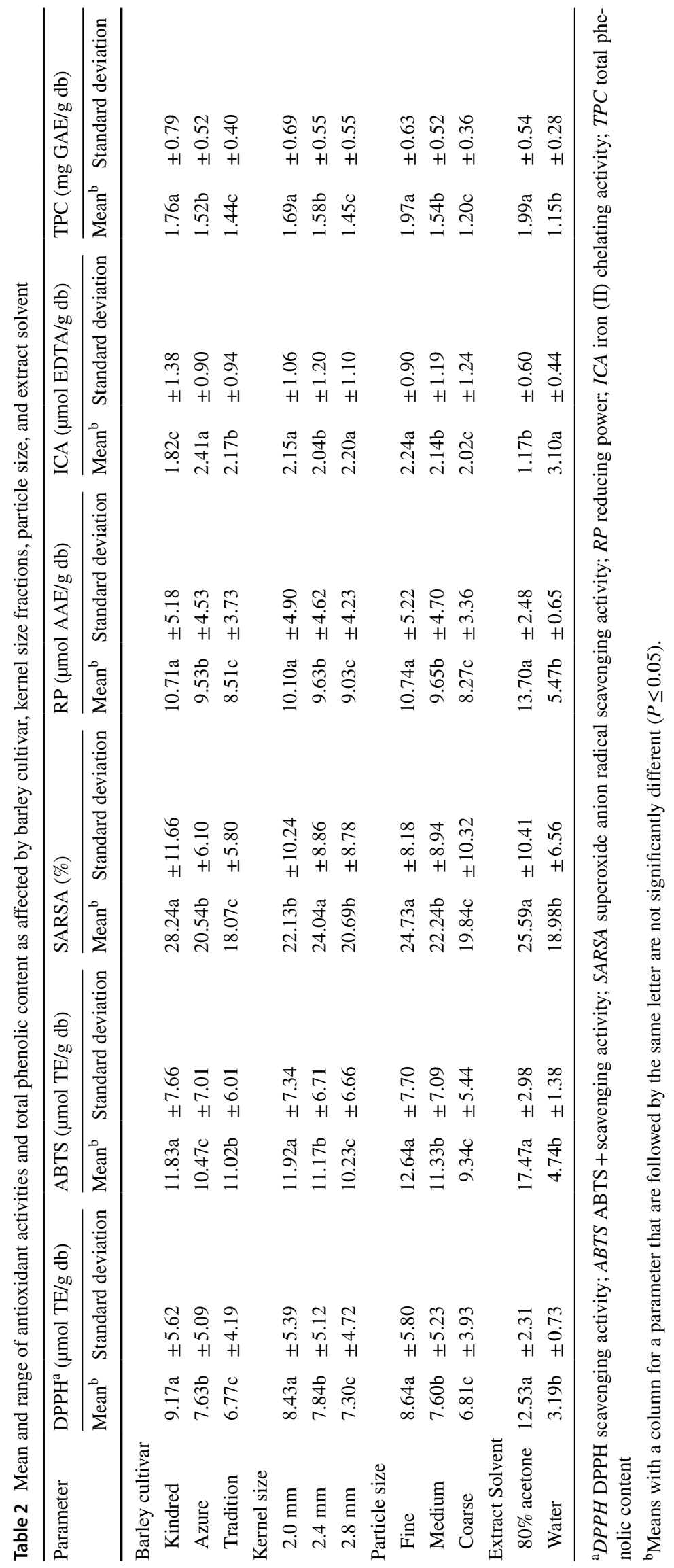


activities and total phenolic content). The associations among total phenolic content and antioxidant activities were determined with Pearson's simple linear correlation. Correlation values were deemed significantly different from zero at $\mathrm{P} \leq 0.01$.

\section{Results}

\section{Effects of solvent, barley variety, kernel size and milling on the antioxidant activities and total phenolic content in barley}

DPPH. Scavenging Activity. DPPH scavenging activity of the barley extract was found to be significantly affected $(\mathrm{P} \leq 0.05)$ by the factors of barley cultivar, kernel size fraction, particle size, and extract solvent (Table 1). Interactions among these four factors were all significant. Examination of Fig. 1a shows that DPPH - radical scavenging activity in three cultivars generally increased with decreasing particle size. The differences in DPPH. scavenging activity between fine and coarse grinding were 1.5 and $2.9 \mu \mathrm{mol} \mathrm{TE} / \mathrm{g} \mathrm{db}$ for Azure and Kindred, respectively, when averaged across kernel size fraction and extract solvent. However, there was no significant difference in DPPH. scavenging activity between fine and medium grinding for the Tradition sample (Fig. 1a). Examination of the interaction between kernel size fraction and extract solvent, shows that DPPH· radical scavenging activity significantly decreased with the increase in kernel plumpness for both the $80 \%$ acetone extract and water extract (Fig. 1b). However, the difference between kernel size fractions became less pronounced when water was used for extraction. Extracts with $80 \%$ acetone extract exhibited $9.3 \mu \mathrm{mol} \mathrm{TE} / \mathrm{g}$ higher than those with water when averaged across barley cultivar, kernel size fractions, and particle size (Table 1). This strongly suggests that $80 \%$ acetone is an efficient solvent for extraction of phenolic components to improve DPPH. scavenging activity.

When averaged across kernel size fractions, particle size, and extract solvent, the DPPH. scavenging activity for Kindred, Azure and Tradition were 9.2, 7.6, and $6.8 \mu \mathrm{mol} \mathrm{TE} / \mathrm{g} \mathrm{db}$, respectively (Table 1). Thinner kernels $(2.0 \mathrm{~mm})$ showed much higher DPPH. scavenging activity than those in the 2.4 and $2.8 \mathrm{~mm}$ fractions when averaged across other factors.

Stepwise linear regression was utilized to determine the relative importance of multiple variables to the overall fit of the data (of independent variables to the dependent variables). As shown in Table 2, extract solvent was able to be alone explained approximately $88 \%$ of the variation in DPPH. scavenging activity observed in the current study. The sequential addition of parameters barley cultivar, particle size, and kernel size increased this to 92,94 , and $95 \%$, respectively. Of all factors studied, extract solvent has the most significant impact (88\%) upon DPPH. scavenging activity, with 12.5 and $3.2 \mu \mathrm{mol} \mathrm{TE} / \mathrm{g}$ db for $80 \%$ acetone and water, respectively, when averaged across other parameters (Table 1).

ABTS + Scavenging Activity. ABTS + scavenging activity was found to be significantly affected $(\mathrm{P} \leq 0.05)$ by all factors (Table 2 ). Interactions among these four factors were all significant. Examination of the interaction of kernel size fraction and particle size (Fig. 2a), shows that ABTS + scavenging activity in three kernel size fractions increased with decreasing particle size and a decrease in plump kernels. However, the differences in ABTS + scavenging activity between fine and coarse grind were less pronounced with increasing kernel plumpness, suggesting
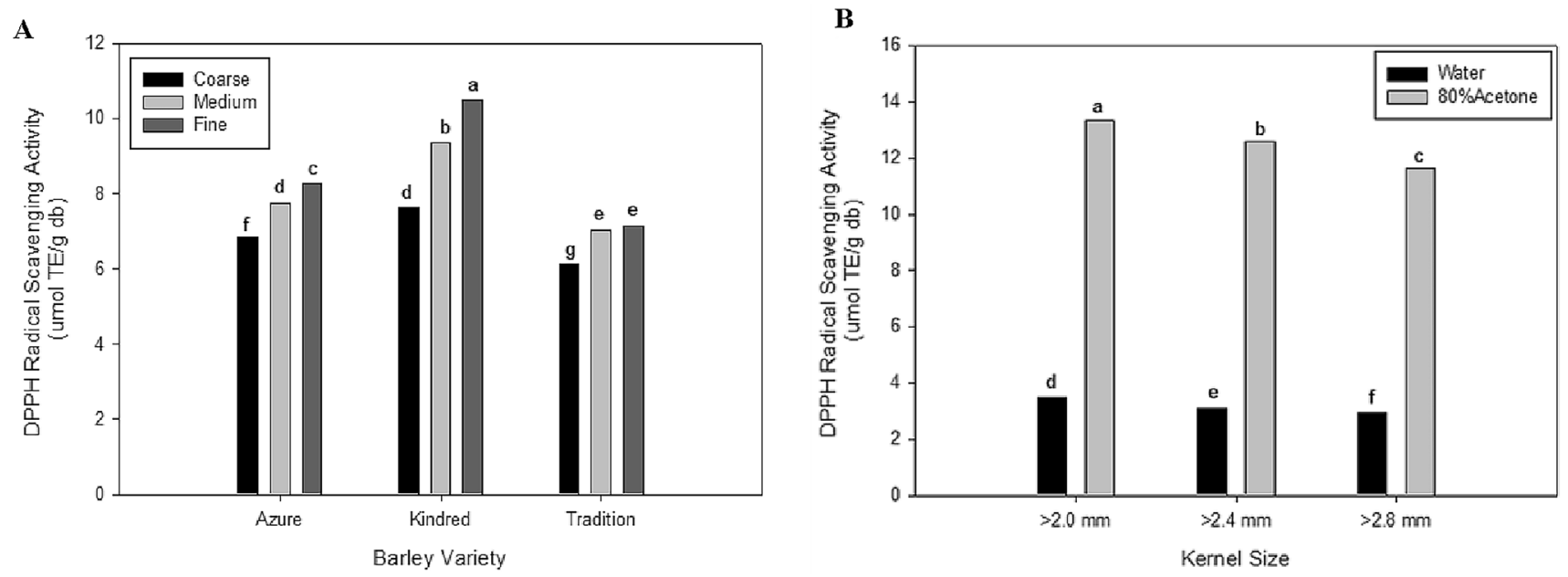

Fig. 1 Interactions for DPPH.Scavenging Activity. a barley cultivar X particle size. b Kernel size fraction X extract solvent. Bars with the same letter are not significantly different at $\mathrm{P} \leq 0.05$ 


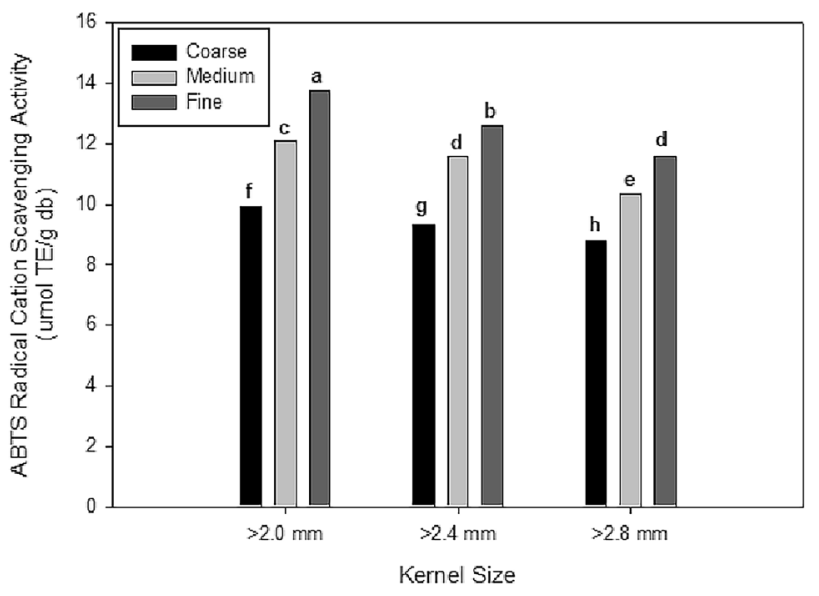

C

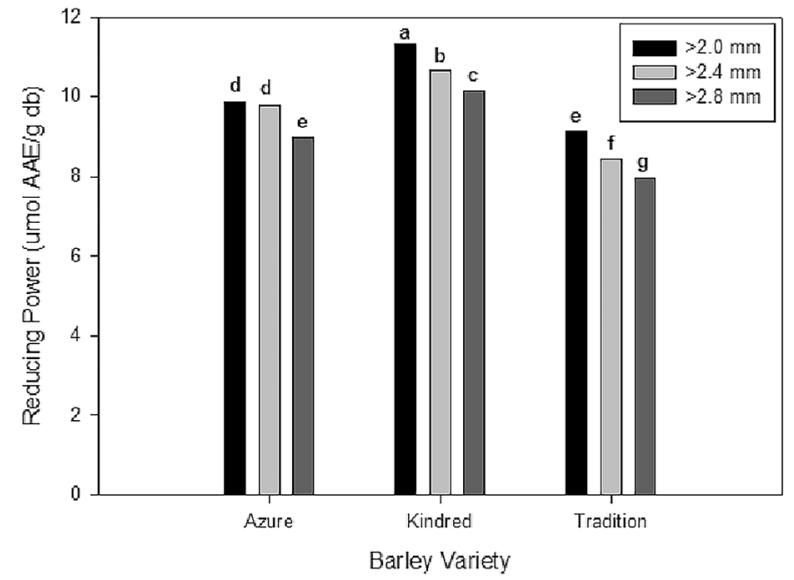

Fig. 2 Interactions for ABTS + Scavenging Activity, Superoxide Anion Radical Scavenging Activity, Iron Chelating Activity. a Kernel size fraction and grind level. b Kernel size fraction and grind level.

that phenolic components associated with ABTS + scavenging activity exist in barley husk and that a finer grinding accentuated this difference.

When averaged across kernel size fractions, grinding, and extract solvent, the highest ABTS + scavenging activity was $11.8 \mu \mathrm{mol} \mathrm{TE} / \mathrm{g} \mathrm{db}$ for Kindred, followed by Tradition $(11.0 \mu \mathrm{mol} \mathrm{TE} / \mathrm{g} \mathrm{db})$ and Azure $(10.5 \mu \mathrm{mol} \mathrm{TE} / \mathrm{g} \mathrm{db})$. When averaged across barley cultivar, particle size, and extract solvent, the ABTS + scavenging activity for the thin kernel size fraction $(2.0 \mathrm{~mm})$ was $11.9 \mu \mathrm{mol} \mathrm{TE} / \mathrm{g} \mathrm{db}$, which was much higher than that in the 2.4 and $2.8 \mathrm{~mm}$ fractions (Table 2).

Like the result from DPPH. scavenging activity, stepwise linear regression showed that extract solvent was clearly the most important factor in the current study, as it alone explained $88 \%$ of the variation observed in ABTS + scavenging activity (Table 3 ). Sequential additions of particle size and kernel size fraction increased the predictive value of the model to 92 , and $93 \%$ respectively. Interestingly, barley cultivar did not pose a significant contribution to the

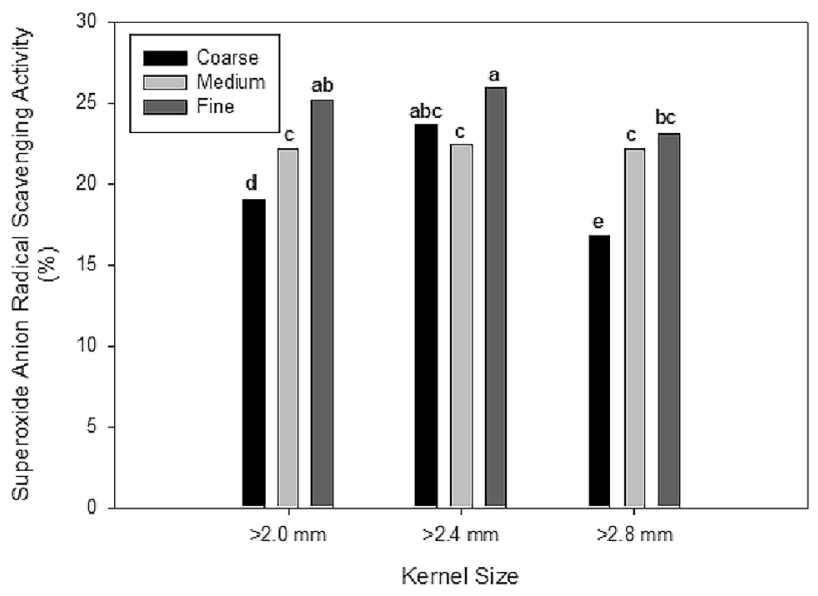

D

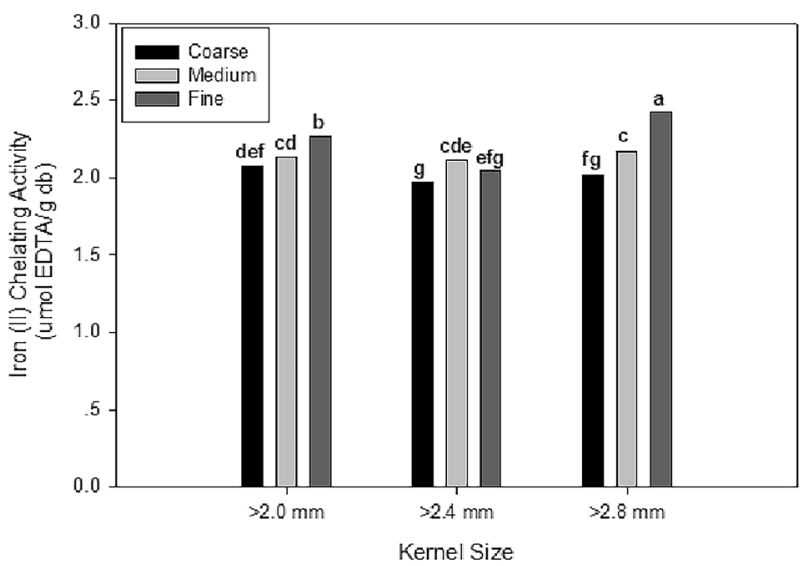

c Barley cultivar and kernel size fraction. d Kernel size fraction and grind level. Bars with the same letter are not significantly different at $\mathrm{P} \leq 0.05$

ABTS + + scavenging activity in the stepwise regression model.

Superoxide Anion Radical Scavenging Activity. All parameters evaluated were found to have a significant effect on superoxide anion radical scavenging activity (Table 3 ). Significant interactions were observed between particle size $\mathrm{x}$ extract solvent, kernel size $\mathrm{x}$ particle size, and barley cultivar $\mathrm{x}$ extract solvent. The interaction of kernel size and particle size shows that superoxide anion radical scavenging activity in three kernel size fractions did not respond uniformly across particle size (Fig. 2b). The trend in the 2.0and $2.8-\mathrm{mm}$ kernel size fractions was for increased superoxide anion radical scavenging activity with finer grinding. This, however, was not observed in the $2.4 \mathrm{~mm}$ kernel size fraction.

When averaged across kernel size, particle size, and extract solvents, the superoxide anion radical scavenging activity for Kindred, Azure and Tradition were 28.2, 20.5, and $18.1 \%$, respectively (Table 2). Interestingly, no significant difference in superoxide anion radical scavenging 


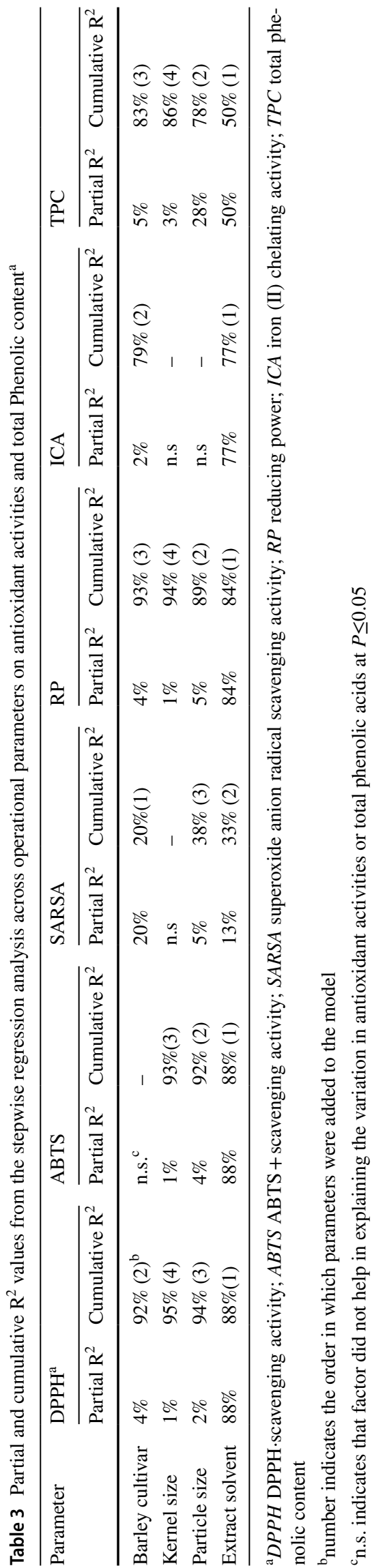

activity was observed between the 2.0 - and $2.8-\mathrm{mm}$ kernel size fractions, but both of the fractions showed lower superoxide anion radical scavenging activity than the $2.4 \mathrm{~mm}$ fraction when averaged across parameters. The trend for the effect of particle size on the superoxide anion radical scavenging activity was similar to that observed for DPPH. and ABTS + scavenging activities, with the finer grindings resulting in higher levels of activity.

Stepwise regression showed that barley cultivar could explain $20 \%$ of the observed variation in superoxide anion radical scavenging activity. Sequential addition of extract solvent and particle size increased this to 33 , and $38 \%$ respectively (Table 3 ). This suggests that superoxide anion radical scavenging activity in barley extracts was more dependent on the barley cultivar.

Reducing Power. Reducing power was found to be significantly affected $(\mathrm{P} \leq 0.05)$ by the factors of barley cultivar, kernel size, particle size, extract solvent. Interactions among these four factors were all significant (Table 2). The interaction between barley cultivar and kernel size (Fig. 2c), showed that for both Kindred and Tradition, higher levels of reducing power were observed with thinner kernel size fraction, but the difference between the 2.0 and $2.4 \mathrm{~mm}$ fractions for Azure was not significant.

As shown in Table 2, reducing power in Kindred, Azure, and Tradition are 10.7, 9.5, and $8.5 \mu \mathrm{mol} \mathrm{AAE} / \mathrm{g} \mathrm{db}$, respectively. Reducing power was also higher with thin kernel size $(10.1 \mu \mathrm{mol} \mathrm{AAE} / \mathrm{g} \mathrm{db})$ than with the plump kernel size $(9.6$ and $9.0 \mu \mathrm{mol} \mathrm{AAE} / \mathrm{g} \mathrm{db}$ for $2.4 \mathrm{~mm}$ and $2.8 \mathrm{~mm}$ fractions, respectively). As expected, the fine grinding yielded higher reducing power than the medium and coarse grindings.

Stepwise linear regression showed that extract solvent was clearly the most important factor for reducing power, as it alone explained $84 \%$ of the variation (Table 3 ). The sequential addition of grinding, barley cultivar, and kernel size increased the model's predictive value to 89,93 , and $94 \%$ respectively.

Iron (II) Chelating Activity. Iron chelating activity was found to be significantly affected $(\mathrm{P} \leq 0.05)$ by all factors (Table 2). Interactions among these four factors were all significant. The examination of the interaction between kernel size and particle size (Fig. 2d), showed that iron chelating activity in three kernel size fractions did not respond uniformly across grind levels. With the $2.8 \mathrm{~mm}$ kernel size fraction, the highest iron chelating activity was observed in the fine grinding, in the $2.0 \mathrm{~mm}$ kernel size fraction, there was no significant difference between medium and coarse grindings. As to the $2.4 \mathrm{~mm}$ kernel size fraction, a significant difference in activity was only observed between medium and coarse grinding.

As shown in Table 2, the iron chelating activity was $2.4 \mu \mathrm{mol}$ EDTA $/ \mathrm{g}$ db for Azure, followed by Tradition $(2.2 \mu \mathrm{mol} \mathrm{EDTA} / \mathrm{g} \mathrm{db})$ and Kindred $(1.8 \mu \mathrm{mol}$ EDTA/g db) 
when averaged across other parameters. No significant difference was observed between the 2.0 and $2.8 \mathrm{~mm}$ kernel size fractions, but both fractions showed higher iron chelating activity than the $2.4 \mathrm{~mm}$ fraction. Extracts with $80 \%$ acetone showed much lower iron chelating activity than those extracted water when averaged across other factors.

Stepwise linear regression showed that extract solvent was still the most important factor for iron chelating activity, as it alone explained $77 \%$ of the variation (Table 3 ). The sequential addition of barley cultivar increased the predictive value of the model to $79 \%$.

Total Phenolic Content (TPC). TPC of the barley extracts was found to be significantly affected $(\mathrm{P} \leq 0.05)$ by the factors of barley cultivar, kernel size, grind, and extract solvent (Table 2). Except for interaction between barley cultivar and kernel size, all other interactions among these four factors were significant. Examination of the interactions between barley cultivar $\mathrm{x}$ particle size and kernel size $\mathrm{x}$ grindings, showed that TPC in three cultivars and three

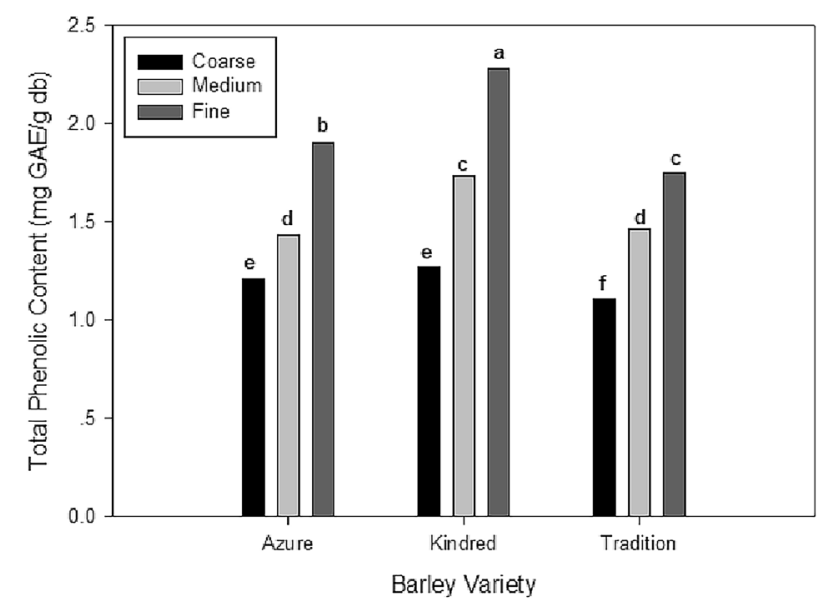

C

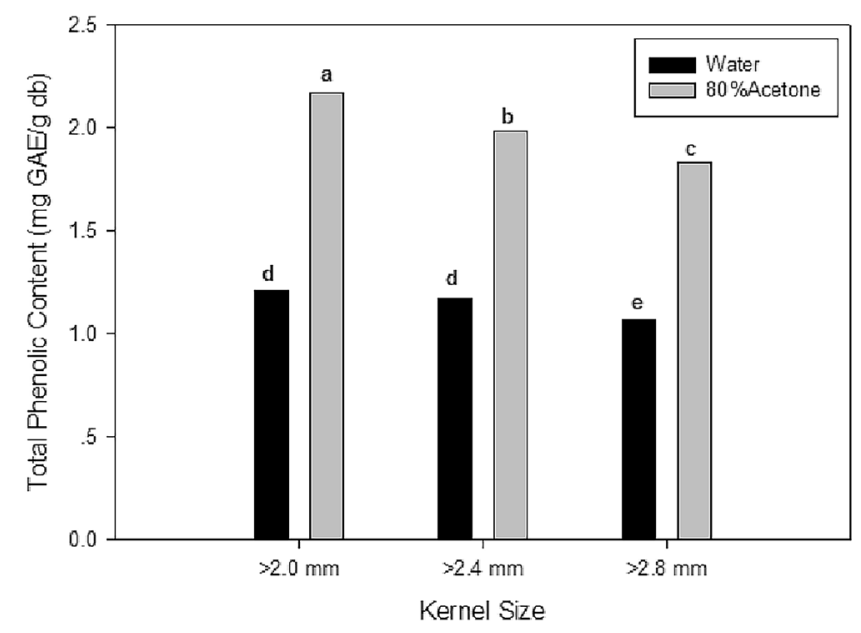

kernel size fractions did respond uniformly across grinding levels (Fig. 3a, b). Total phenolic contents generally increased with decreasing particle size. Examination of the interaction of kernel size and extract solvent (Fig. 3c), shows that TPC in three kernel size fractions decreased with plumper kernel size fraction regardless of extract solvent. However, the differences in TPC between $80 \%$ acetone and water extracts were less pronounced in the plump kernel fractions.

When averaged across kernel size, grind, and extract solvent, the TPC for Kindred, Azure and Tradition were 1.8, 1.5 , and $1.4 \mathrm{mg} \mathrm{GAE} / \mathrm{g} \mathrm{db}$, respectively (Table 2). Thin kernels $(2.0 \mathrm{~mm})$ showed slightly TPC $(1.7 \mathrm{mg} \mathrm{GAE} / \mathrm{g} \mathrm{db})$ than the 2.4 and $2.8 \mathrm{~mm}$ fractions ( 1.6 and $1.5 \mathrm{mg} \mathrm{GAE} / \mathrm{g} \mathrm{db}$, respectively) when averaged across other parameters. When averaged, $80 \%$ acetone extracts showed much higher TPC than those prepared with water. This suggests that acetone can efficiently facilitate the extraction of phenolic components from barley grain.

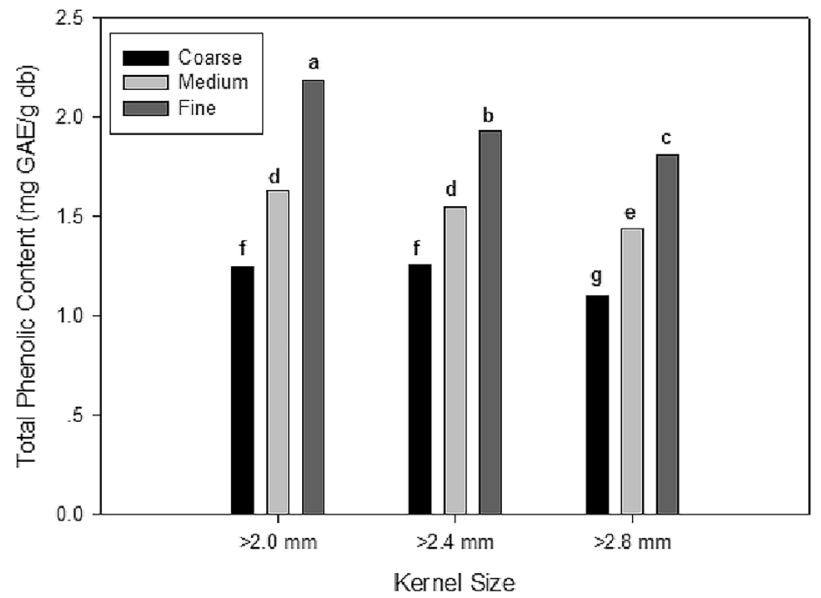

Fig. 3 Interactions for Total Phenolic Content. a Barley cultivar and grinding level. b Kernel size fraction and grinding level. c Kernel size fraction and extract solvent. Bars with the same letter are not significantly different at $\mathrm{P} \leq 0.05$ 
As shown in Table 3, extract solvent alone was able to explain approximately $50 \%$ of the variation in TPC observed in the current study. Sequential addition of the parameters, grinding, cultivar, and kernel increased this to 78,83 , and $86 \%$, respectively. Of all factors studied, extract solvent and grinding were the major factors affecting the TPC.

\section{Correlations between total phenolic content and antioxidant activities}

Correlation analysis between TPC and antioxidant activities was performed across all the samples in the current study and the results shown in Table 4. All correlation values between TPC and antioxidant activities were found to be significantly different $(\mathrm{P} \leq 0.01)$. In the current study, strong correlations were observed between TPC and DPPH-activity $(r=0.86)$, and ABTS + scavenging activity $(r=0.85)$ and reducing power $(r=0.89)$. These are in agreement with the report of Zhao et al. [37] for Chinese barley varieties and Zhou et al. [32] for wheat bran. There was a weak but still significant association between TPC and superoxide anion radical scavenging $(r=0.59)$, while a negative significant correlation was observed between TPC and iron chelating activity $(\mathrm{r}=-0.62)$. Relatively strong correlations $(r=0.97-0.98)$ were observed among DPPH $\&$ $\mathrm{ABTS}+$ scavenging activities and reducing power.

This indicates that these three antioxidant activity traits (DPPH., ABTS + scavenging activities and reducing power) above can express in the similar antioxidant activity trend associated with TPC in the barley extracts (Table 4). Negative but significant associations were found between iron chelating activity and other four antioxidant activity traits. Superoxide anion radical scavenging showed weak but significant correlations with the other two antioxidant activity traits.

\section{Effects of solvent, barley variety, kernel size and milling on phenolic acid composition in barley}

The phenolic acid compositions across the barley variety, kernel size fractions, grinding level, and extract solvent are shown in Table 5. In terms of barley variety, there was no significant difference in gallic acid, vanillic acid, p-coumaric acid and ferulic acid when averaged across other factors. Azure and Tradition showed almost twice the protochuric acid content than Kindred, while the sum of syringic acid and caffeic acid in Kindred showed much higher than those in Azure and Tradition. As to kernel size fractions, all the individual phenolic acid showed the highest values in thin kernel size fraction $(2.0 \mathrm{~mm})$ when averaged across barley other parameters. This can be explained in that thin kernels have a larger proportion of husk than plump kernels. However, there were no significant differences in individual phenolic acids two plumper kernel size fractions (2.4 and $2.8 \mathrm{~mm}$ ). The influence of particle size was used to evaluate the grain matrix effects on the extraction of phenolic acids. As expected, fine grinding showed the highest levels for all the individual phenolic acid when averaged across other factors. There were no significant differences in contents of protochuric acid, p-coumaric acid and ferulic acid between medium and coarse factions. However, significant differences were detected in gallic acid, vanillic acid, and the sum of syringic acid and caffeic acid for medium and coarse grindings. But the response was not uniform across the medium and coarse fractions. Extract solvent has a significant effect on the phenolic acid compositions in that $80 \%$ acetone tended to favor the extraction of all the individual phenolic acids when averaged across other factors.

Stepwise linear regression analysis was sequentially performed to evaluate the contribution of individual phenolic acid on the different antioxidant traits. As shown in Table 6, the sum of syringic acid and caffeic acid was able to explain approximately $89 \%$ of the variation in DPPH. scavenging activity observed in the current study. The sequential addition of parameters gallic acid and p-coumaric acid increased the model to 91 and $92 \%$, respectively. As to
Table 4 Correlations analysis between barley antioxidant activities and total phenolic contents

\begin{tabular}{lllllrr}
\hline & DPPH & ABTS & SARSA & RP & ICA & TPC \\
\hline DPPH & 1 & $0.98^{*}$ & $0.47^{*}$ & $0.99^{*}$ & $-0.83^{*}$ & $0.86^{*}$ \\
ABTS & & 1 & $0.41^{*}$ & $0.97^{*}$ & $-0.80^{*}$ & $0.85^{*}$ \\
SARSA & & 1 & $0.48^{*}$ & $-0.53^{*}$ & $0.59^{*}$ \\
RP & & & 1 & $-0.80^{*}$ & $0.89^{*}$ \\
ICA & & & & & 1 & $-0.62^{*}$ \\
TPC & & & & & & 1 \\
\hline
\end{tabular}

$D P P H$ DPPH scavenging activity; ABTS ABTS + scavenging activity; SARSA superoxide anion radical scavenging activity; $R P$ reducing power; $I C A$ iron (II) chelating activity; $T P C$ total phenolic content

*Significant at the $P \leq 0.01$ level of probability 


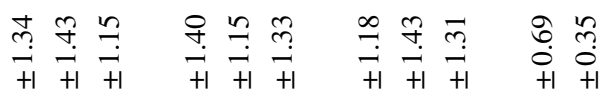

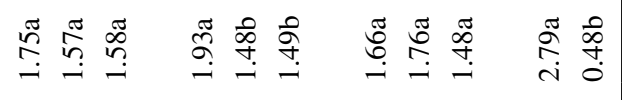

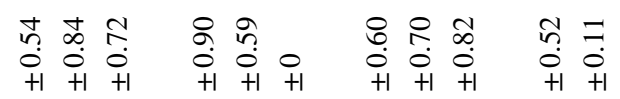

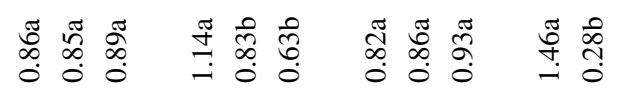

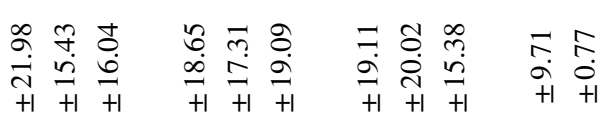

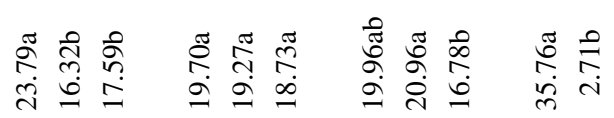

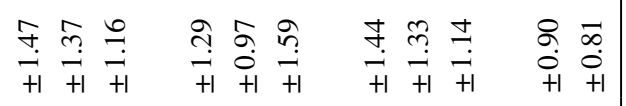

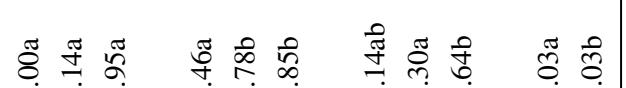
लं ते लेत

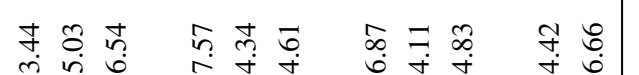

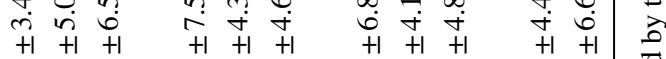

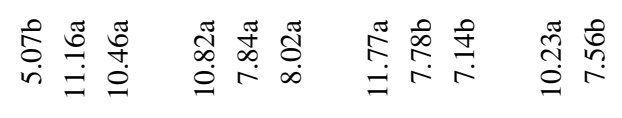

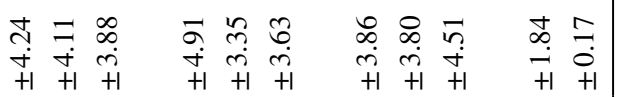

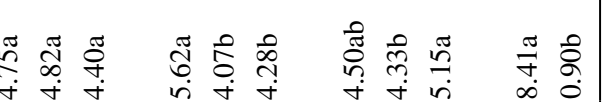

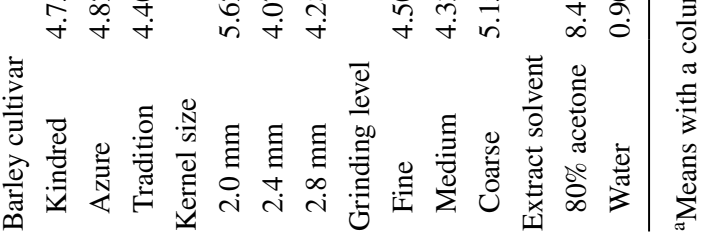




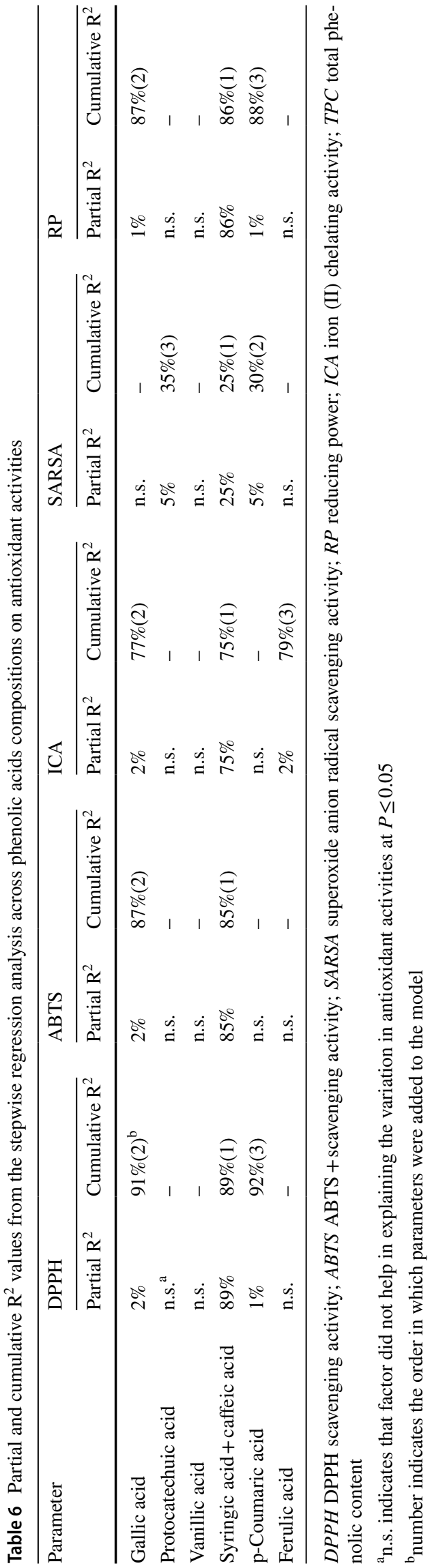

ABTS + scavenging activity, the sum of syringic acid and caffeic acid was able to explain approximately $85 \%$ of the variation. Sequential addition of gallic acid increased the model to $87 \%$. Like the DPPH. scavenging activity and ABTS + scavenging activity, the sum of syringic acid and caffeic acid exhibited the major contribution to the reducing power and iron chelating activity, explaining $86 \%$ and $75 \%$ of the variations, respectively. However, unlike the other four antioxidant traits, only $25 \%$ of the variation in the superoxide anion radical scavenging activity can be explained by the sum of syringic acid and caffeic acid. Sequential addition of p-coumaric acid and protochuric acid increased the predictive value of the model to 30 and $35 \%$ respectively. It suggested that the superoxide anion radical scavenging activity in the barley cannot be intensively explained by the phenolic acid compounds.

\section{Discussion}

The current study evaluated the impact of cultivar, kernel size, grind particle size and extraction solvent on antioxidant activities, TPC and phenolic acids in barley. Many of the results are confirmatory of previous studies. This is especially the case in terms of cultivar effects, where our previous study [17], as well as those of other researchers [18-21] have demonstrated the genotype of malting barley to have significant effects on the TPC and antioxidant activities (including DPPH, ABTS +, RP, and ICA).

Kernel size was also found to have a significant impact on all antioxidant activities and TPC when averaged over cultivars and the other parameters. As we had previously demonstrated [17], the thinnest kernels $(2.0 \mathrm{~mm})$ generally had higher levels of antioxidant activities and TPC. Exceptions were SARSA and ICA. However, examination of the data in Tables 2 and 5 shows that numeric differences in these parameters were quite small. In terms of individual phenolic acids, the contents in thin kernel $(2.0 \mathrm{~mm}) \mathrm{had}$ almost double the contents of the plumpest kernels $(2.8 \mathrm{~mm})$ with exceptions of ferulic and p-coumaric acids. It indicated that barley with thin kernel size contained higher phenolic acids, which can be due to more husk in thin barley.

Not surprisingly, extraction solvent was found to have the largest effect for most antioxidant activities. Zhao et al. [27] had previously reported that the highest DPPH. and ABTS + radical scavenging activities were found when barley was extracted with $80 \%$ acetone, when compared to $80 \%$ methanol and water extracts. Extracts with $80 \%$ acetone also were found to have the greatest reducing power as well as highest content of a number of phenolic including ferulic and p-coumaric acids.

However, in brewing, the extraction of phenolics from grains occurs in the mashing and lautering processes, when 
the water solvent is in contact with the grist [38]. Boiling may result in reactions such as the polymerization and precipitation of phenolic. Results of the current and previous studies, that have evaluated solvent, thus suggest that a lesser portion of the total DPPH - and ABTS + radical scavenging, reducing power, and free phenolic acids are extracted in brewing. For example, in the current study water extracts had only about $25 \%$ of the DPPH. and ABTS + radical scavenging activities that were found in the acetone extracts. However, it must also be remembered that bound phenolic forms can be enzymatically converted to free phenolic acids during malting and mashing.

The unique aspect of the current study was the examination of the effect of grind particle size. The particle size distribution of the three fractions is shown in Table 1. The finest grind had approximately 61 and $25 \%$ of the sample retained on sieves with 0.250 - and $0.149-\mathrm{mm}$ openings, respectively. With the coarsest grind, approximately 61 and $34 \%$ of the samples were retained sieves with 0.595 - and 0.250 -mm openings, respectively While the medium grind was intended to be intermediate, the particle size distribution was actually closer to that of the coarse grind. All are somewhat finer than would be seen in brewer's grist for a lauter tun.

Grinding had a significant impact on TPC and all antioxidant assays, with decreasing particle size resulting in increased TPC and antioxidant activity (Table 2). These results are similar to those reported by Luthria et al. [9] and Zhou et al. [32] for wheat bran. It can be explained by that phenolic compounds in finer particles exhibited to be more extractable by solvent in ease. With the exception of iron chelating activity, where a minimal difference was observed, the fine grind generally resulted in about $20 \%$ more activity than that found in the coarser grind. However, in Table 5, grind particle size did not show much difference for individual phenolic acids. Moore et al. [39] reported three basic states of phenolic acids in wheat, including less than $9 \%$ of soluble conjugated form, $1 \%$ of soluble free form and $90 \%$ of insoluble bound form. The extraction response of phenolic acids might not be uniform, accounting for free and extractable individuals.

\section{Conclusions}

The parameters evaluated in the current study, barley cultivars, kernel size, grind particle size, and extraction solvent, all showed significantly influences on antioxidant activity (including DPPH - radical scavenging activity, ABTS + scavenging activity, superoxide anion radical scavenging activity, reducing power, Iron chelating activity) and total phenolic content.
(1). Extraction solvent ( $80 \%$ acetone) was the most important factor to affect the antioxidant activities, such as reducing power iron chelating activity and total phenolic content across the study. Grinding was another important factor affecting the total phenolic content, while cultivar was the factor to significantly affect SARSA.

(2). All correlation values between TPC and antioxidant activities were found to be significantly different $(\mathrm{P} \leq 0.01)$. Strong correlations were observed between TPC and DPPH scavenging activity $(\mathrm{r}=0.86)$, ABTS + scavenging activity $(r=0.85)$ and reducing power $(r=0.89)$. The correlations among DPPH. scavenging activity, ABTS + scavenging activities, and reducing power were investigated by $r=0.97-0.98$, while the correlations between TPC and SARSA $(r=0.59)$, TPC and ICA $(r=-0.62)$ were weak and negatively low, respectively.

$3)$. Although barley varieties and milling showed obvious influences on phenolic compounds, and fine grinding obtained the highest levels for all the individual phenolic acids, the influences were not uniformly distributed. Linear regression analysis indicated that SA (syringic acid) and CA (caffeic acid) offered the main contribution to antioxidant activities (DPPH, ABTS, RP and ICA) and did not influence SARSA significantly.

Acknowledgements The authors wish to express their appreciation to American Malting Barley Association, Inc. for financial support of this research.

Author contributions YL investigation and statistical analysis; BZ writing — review and editing; ZJ writing — review and formating; PS supervision and editing. All authors have read and agreed to the published version of the manuscript.

\section{Declarations}

Conflict of interest The authors declare no conflict of interest. The funders had no role in the design of the study; in the collection, analyses, or interpretation of data; in the writing of the manuscript, or in the decision to publish the results.

Open Access This article is licensed under a Creative Commons Attribution 4.0 International License, which permits use, sharing, adaptation, distribution and reproduction in any medium or format, as long as you give appropriate credit to the original author(s) and the source, provide a link to the Creative Commons licence, and indicate if changes were made. The images or other third party material in this article are included in the article's Creative Commons licence, unless indicated otherwise in a credit line to the material. If material is not included in the article's Creative Commons licence and your intended use is not permitted by statutory regulation or exceeds the permitted use, you will need to obtain permission directly from the copyright holder. To view a copy of this licence, visit http://creativecommons.org/licenses/by/4.0/. 


\section{References}

1. J.W. Anderson, T.J. Hanna, X.J. Peng, R.J. Kryscio, Whole grain foods and heart disease risk. J. Am. Coll. Nutr. 19, 291s-299s (2000)

2. G.A. Soliman, Dietary fiber, atherosclerosis, and cardiovascular disease. Nutrients 11, 1155 (2019)

3. S.M. Liu, J.E. Manson, M.J. Stampfer, F.B. Hu, E. Giovannucci, G.A. Colditz, C.H. Hennekens, W.C. Willett, A prospective study of whole-grain intake and risk of type 2 diabetes mellitus in US women. Am. J. Public Health 90, 1409-1415 (2000)

4. J. Montonen, R. Jarvinen, M. Heliovaara, A. Reunanen, A. Aromaa, P. Knekt, Food consumption and the incidence of type II diabetes mellitus. Eur. J. Clin. Nutr. 59, 441-448 (2005)

5. J.L.M. Andersen, L. Hansen, B.L.R. Thomsen, L.R. Christiansen, L.O. Dragsted, A. Olsen, Pre- and post-diagnostic intake of whole grain and dairy products and breast cancer prognosis: the Danish diet, cancer and health cohort. Breast Cancer Res. Treat. 179, 743-753 (2020)

6. C.M. Kasum, D.R. Jacobs, K. Nicodemus, A.R. Folsom, Dietary risk factors for upper aerodigestive tract cancers. Int. J. Cancer 99, 267-272 (2002)

7. N. Okarter, R.H. Liu, Health benefits of whole grain phytochemicals. Crit. Rev. Food Sci. Nutr. 50, 193-208 (2010)

8. A. Gani, S.M. Wani, F.A. Masoodi, G. Hameed, Whole-grain cereal bioactive compounds and their health benefits: a review. J. Food Process Technol. 3, 146 (2012). https://doi.org/10.4172/ 2157-7110.1000146

9. D.L. Luthria, J. Lu, K.M. Maria John, Bioactive phytochemicals in wheat: extraction, analysis, processing, and functional properties. J. Funct. Foods 18, 910-925 (2015)

10. H. Zieliński, H. Kozłowska, Antioxidant activity and total phenolics in selected cereal grains and their different morphological fractions. J. Agric. Food Chem. 2000(48), 2008-2016 (2000)

11. E. Weichselbaum, J.L. Buttriss, Polyphenols in the diet. Nutr. Bull. 35, 157-164 (2010)

12. C. Fares, C. Platani, A. Baiano, V. Menga, Effect of processing and cooking on phenolic acid profile and antioxidant capacity of durum wheat pasta enriched with debranning fractions of wheat. Food Chem. 119, 1023-1029 (2010)

13. R.H. Liu, Potential synergy of phytochemicals in cancer prevention: mechanism of action. J. Nutr. 134, 3479S-3485S (2004)

14. H. Gencoglu, C. Orhan, K. Sahin, Phytochemical therapies in vascular functioning: a molecular approach. Curr. Vasc. Pharmacol. 15, 327-338 (2017)

15. F.A. Tomás-Barberán, C. Andrés-Lacueva, Polyphenols and health: current state and progress. J. Agric. Food Chem. 60, 8773-8775 (2012)

16. S. McGuire, US Department of Agriculture and US Department of Health and Human Services, Dietary Guidelines for Americans, 2010 7th edition Washington, DC: US Government Printing Office January 2011. Adv. Nutr. 2, 293-2949 (2011)

17. B. Zhou, Z. Jin, P. Schwarz, Y. Li, Impact of genotype, environment, and malting conditions on the antioxidant activity and phenolic content in US malting barley. Fermentation 6(2), 48 (2002). https://doi.org/10.3390/fermentation6020048

18. S. Narwal, D. Kumar, R.P.S. Verma, Effect of genotype, environment and malting on the antioxidant activity and phenolic content of Indian barley. J. Food Biochem. 40, 91-99 (2016)

19. C. Almaguer, C. Scho“nberge, M. Gastl, E.K. Arendt, T. Becker, Humulus lupulus-a story that begs to be told. A review. J. Inst. Brew. 120, 289-314 (2014)

20. T. Madhujith, M. Izydorczyk, F. Shahidi, Antioxidant properties of pearled barley fractions. J. Agric. Food Chem. 54, 3283-3289 (2006)
21. C. Liyana-Pathirana, S. Fereidoon, Importance of insoluble-bound phenolics to antioxidant properties of wheat. J. Agric. Food Chem. 54, 1256-1264 (2006)

22. M. Blandino, V. Sovrani, F. Marinaccioa, A. Reyneri, L. Rolle, S. Giacosa, M. Locatelli, M. Bordiga, F. Travaglia, J.D. Coïsson, M. Arlorio, Nutrition and technological quality of bread enriched with an intermediated pearled wheat fraction. Food Chem. 141, 2549-2557 (2013)

23. A.K. Holtekjølen, C. Kinitz, S.H. Knutsen, Flavanol and bound phenolic acid contents in different barley varieties. J. Agric. Food Chem. 54, 2253-2260 (2006)

24. S. Cai, Z. Han, Y. Huang, Z.-H. Chen, G. Zhang, F. Dai, Genetic diversity of individual phenolic acids in barley and their correlation with barley malt quality. J. Agric. Food Chem. 63, 7051-7057 (2015)

25. B. Verma, P. Hucl, R.N. Chibbar, Phenolic acid composition and antioxidant capacity of acid and alkali hydrolysed wheat bran fractions. Food Chem. 116, 947-954 (2009)

26. D.L. Luthria, Y. Lu, K.M. Maria John, Bioactive phytochemicals in wheat: Extraction, analysis, processing, and functional properties. J. Funct. Foods 18, 910-925 (2015)

27. H.F. Zhao, J.J. Dong, J. Lu, J. Chen, Y. Li, L.J. Shan, Y. Lin, W. Fan, G.X. Gu, Effects of extraction solvent mixtures on antioxidant activity evaluation and their extraction capacity and selectivity for free phenolic compounds in barley. J. Agric. Food Chem. 54, 7277-7286 (2006)

28. T.I. Lafka, V. Sinanoglou, E.S. Lazos, On the extraction and antioxidant activity of phenolic compounds from winery wastes. Food Chem. 104, 1206-1214 (2007)

29. A. Ismail, Z.M. Marjan, C.W. Foong, Total antioxidant activity and phenolic content in selected vegetables. Food Chem. 87, 581-586 (2004)

30. N. Pellegrini, M. Serafini, B. Colombi, D. Del Rio, S. Salvatore, M. Bianchi, F. Brighenti, Total antioxidant capacity of plant foods, beverages and oils consumed in Italy assessed by three different in vitro assays. J. Nutr. 133, 2812-2819 (2003)

31. B.J. Xu, S.K.C. Chang, A comparative study on phenolic profiles and antioxidant activities of legumes as affected by extraction solvents. J. Food Sci. 72, S159-S166 (2007)

32. K.Q. Zhou, L.L. Yu, Effects of extraction solvent on wheat bran antioxidant activity estimation. LWT-Food Sci. Technol. 37, 717-721 (2004)

33. M. Xu, Z. Jin, J.B. Ohm, P. Schwarz, J. Rao, B. Chen, Improvement of the antioxidative activity of soluble phenolic compounds in chickpea by germination. J. Agric. Food Chem. 66, 6179-6187 (2018)

34. M. Oktay, I. Gulcin, O.I. Kufrevioglu, Determination of in vitro antioxidant activity of fennel (Foeniculum vulgare) seed extracts. LWT-Food Sci. Technol. 36, 263-271 (2003)

35. J. Yu, T. Vasanthan, F. Temelli, Analysis of phenolic acids in barley by high-performance liquid chromatography. J. Agric. Food Chem. 49, 4352-4358 (2001)

36. M.J. Crawley, The R Book (Wiley, Chichester, 2007), pp. 511-526

37. H.F. Zhao, W. Fan, J.J. Dong, J. Lu, J. Chen, L.J. Shan, Y. Lin, W.B. Kong, Evaluation of antioxidant activities and total phenolic contents of typical malting barley varieties. Food Chem. 107, 296-304 (2008)

38. J. Wannenmacher, M. Gastl, T. Becker, Phenolic substances in beer: structural diversity, reactive potential and relevance for brewing process and beer quality. Compr. Rev. Food Sci. Food Saf. 17, 953-988 (2018)

39. J. Moore, Z. Hao, K. Zhou, M. Luther, J. Costa, L. Yu, Carotenoid, tocopherol, phenolic acid, and antioxidant properties of Marylandgrown soft wheat. J. Agric. Food Chem. 53, 6649-6657 (2005) 
Publisher's Note Springer Nature remains neutral with regard to jurisdictional claims in published maps and institutional affiliations. 